\title{
MANUEL COLMEIRO \\ Y LA PROPIEDAD DE LA TIERRA \\ EN GALICIA
}

\author{
XOSE CORDERO TORRON
}

Universidad de Santiago de Compostela

Manuel Colmeiro Penido fue el primer economista gallego en vislumbrar la vía que debía de seguir la agricultura gallega para su modernización. Era ésta una vía de ruptura con la herencia del Antiguo Régimen y con la forma en que se desarrollaba en Galicia la revolución liberal.

En la Galicia del Antiguo Régimen la figura jurídica predominante en la articulación de las relaciones entre los rentistas, propietarios agrarios, y los campesinos era el foro '. En las relaciones sociales articuladas por esta figura jurídica existía una divergencia de intereses entre foristas y subforistas, facciones ambas de la clase dominante gallega. Una divergencia que era una consecuencia de la existencia de una contradicción surgida en torno a la participación que cada una de ellas tenía, o habría de tener en el futuro, en la apropiación del excedente agrícola. Debido a ello, va a existir una fuerte polémica que conduciría a que se dictase la Real provisión de 11 de mayo de 1763 , en la que se recogían las alegaciones de los subforistas y se ordenaba, si bien mientras no se adoptase una resolución definitiva que, por otra parte, nunca llegaría, la renovación obligatoria de los foros «con el canon y pensión que actualmente y hasta ahora han satisfecho a los dueños» ${ }^{2}$.

En las primeras etapas de la revolución burguesa en España, las Cortes de Cádiz y el Trienio Liberal, no se produjeron modificaciones sustanciales, a no ser las desamortizadoras, con respecto a la situación en la que estaban los foros tras la Real Provisión de $1763^{3}$, y así se llega a 1836, cuando en la revolución liberal se hubo de plantear de nuevo en las leyes de desamortización, de abolición de señoríos y en los primeros proyectos de Código Civil la conveniencia de confirmar o de anular las figuras jurídicas que, como el foro, tenian un origen señorial.

' García Lombardero (1973); Barreiro Mallón (1973); Pérez García (1979); Saavedra (1979); Saavedra (1985); Villares (1982).

${ }_{2}$ La Real Provisión de 11 de mayo de 1763 fue publicada más tarde en Revista Jurí. dica y Administrativa de Galicia, tomo I, 1852, pp. 303-306; García Lombardero y Dopico (1976).

${ }^{3}$ Cordero Torrón (1986). 
En la ley de 26 de agosto de 1837 se rectifican las anteriores leyes de señoríos para asegurar a foristas y subforistas la permanencia de sus «contratos» ${ }^{4}$. En la desamortización se opta por favorecer las enajenaciones en subasta pública en lugar de las redenciones de rentas forales por los utilitarios ${ }^{5}$. En el proyecto de Código Civil de 1836, que pronto sería retirado, se exceptuaban los foros de Galicia y las enfiteusis de la Corona de Aragón de la regularización de los censos que en él se establecía, remitiéndolos a una futura ley especial, en tanto que para los restantes censos se afirmaba su redimibilidad, se otorgaba el derecho de tanteo a los dos dominios y se suprimía el laudemio en las enfiteusis futuras, reduciéndolo en las ya existentes al 2 por 100 del precio de venta ${ }^{6}$.

Manuel Colmeiro critica en 1843 la vía seguida por la revolución burguesa en Galicia y plantea en su Memoria sobre el modo más acertado de remediar los males inberentes a la extremada subdivisión de la propiedad territorial en Galicia una vía alternativa que no deja por ello de inscribirse dentro de la lógica de la economía liberal.

Para Colmeiro, el desarrollo de la agricultura gallega pasaba por la consolidación en el «utilitario» de los dos dominios forales y por el aprovechamiento privado de los comunales ${ }^{7}$.

El problema más serio con el que se enfrentaba la opción de Colmeiro estaba en la carencia de un sustrato social que apoyase sus tesis. Descartados foristas y subforistas, interesados en el mantenimiento del régimen foral, la burguesía gallega, que había manifestado en repetidas ocasiones su apoyo al régimen liberal ${ }^{8}$, tampoco era partidaria de la vía alternativa propuesta por Colmeiro. La contradicción entre la opción liberal de Colmeiro y la también liberal de la burguesía gallega, o al menos de un sector importante

- En el artículo 10 de la Ley de 26 de agosto de 1837 se dice: «Cuando los predios que fueren de señorío se hayan dado a foro, censo o enfiteusis, aunque el señorío sea reversible o incorporable a la nación, continuará el dominio útil en los que lo hayan adquirido, considerándose como propiedad particular. Los contratos que se hayan celebrado después de la primera concesión para transferir a otras manos los foros, censos y enfiteusis, se cumplirán como hasta ahora y según su tenor," Véanse García Ormaechea (1932) y Moxo (1965).

${ }^{5}$ Cordero Torrón (1986); Carmona Badía y Cordero Torrón (1980).

- Lasso Gaite (1979).

' Sobre los bienes comunales dice que: «Acotando, ni el ganado quedaria sin pastos, ni el labrador sin combustible para su hogar, si pastos y combustible podemos llamar el miserable esquilmo que los montes producen abandonados a sí mismos, y aun a despecho de la mano del hombre en constante lucha con la naturaleza, porque por la sola razón de que nadie se encariña con la tierra que se dice pertenecer a todos, nadie tampoco la defiende, ni los terrenos se utilizan, sino que se talan hasta desterrar de ellos el menor vestigio de vegetación», y sobre los foros, que «... la consolidación del dominio debe ser el primer paso del legislador interesado en reorganizar nuestra constitución agrícola...». Colmeiro (1843), pp. 23 y 47.

Alonso Alvarez (1986). 
de esta burguesía, se evidencia más que en ningún otro punto en la opinión que para ambos merecía la desamortización tal como se estaba efectuando.

Colmeiro, partidario de la redención foral y de que coincidiesen en un mismo individuo la propiedad y el cultivo de las tierras, juzgaba que la desamortización no había influido en mejorar la suerte de los labradores y tan sólo había beneficiado a un cortísimo número de agiotistas.

«... no influyó hasta aquí de un modo sensible en mejorar la suerte de la clase labradora, ni apenas influirá tampoco en lo futuro, a no hacerse todavía más difícil su adquisición (de los Bienes Nacionales). Dígase sino si los labradores compraron muchos Bienes Nacionales, o si al contrario no se convirtió la desamortización en provecho exclusivo de un cortísimo número de agiotistas...» ${ }^{9}$.

Frente a ello, defendía la enajenación a censo de parte de los Bienes Nacionales, estipulándose un canon en función del producto neto de las fincas enajenadas, que sería redimible a voluntad del censualista ${ }^{10}$. Por otra parte, los bienes comunales serían objeto de reparto entre los vecinos y tendrían un aprovechamiento privado ${ }^{11}$.

La burguesía comercial gallega hacía en cambio una alabanza de la desamortización en uno de sus órganos oficiosos de comunicación ${ }^{12}$, y no ponía en cuestión el régimen foral. En el Boletín Mercantil e Industrial de Galicia de la ciudad de La Coruña se dice que «Nosotros consideramos la desamortización civil y eclesiástica en España, como la más importante de las conquistas de su revolución. Grandes capitales, inmensos terrenos, numerosos edificios, pasaron al dominio particular; esto es, cesaron de ser improductivos para ser productivos» ${ }^{13}$.

En las reflexiones del «Boletín» se silenciaba que en Galicia la mayor parte de las enajenaciones de Bienes Nacionales eran de foros, de los que tan sólo se enajenaba el «dominio directo», y ni siquiera se mencionaban los

${ }^{9}$ Colmeiro (1843), pp. 20.

${ }^{10}$ Colmeiro (1843), p. 21.

11 Propugna que se acordase una ley que ordenase que «dentro de un plazo dado y largo, cuanto fuere preciso, todos los baldíos de cada común o parroquia (que es el distrito rural de uso más frecuente) se repartiesen entre los vecinos y se pusiesen en cultivo, - a lo menos se acotasen, añadiendo que si corrido el término señalado no lo tuviesen hecho, los baldíos serían declarados propiedad del estado para que el gobierno los vendiese o adjudicase a nuevos pobladores, o en fin dispusiese de ellos, según se acordare á lo sucesivom. Colmeiro (1843), p. 24.

${ }_{12}$ Sobre el periódico Boletín Mercantil e Industrial de Galicia y su papel de órgano oficioso de la burguesía comercial coruñesa, véase Rodríguez Galdó (1981).

${ }^{13}$ Boletin Mercantil e Industrial de Galicia (1848). 
problemas que podía suscitar la subsistencia de los foros tras la revolución liberal. Es más, parece como si la polémica foral le fuera ajena a la burguesía gallega. Pero el absentismo de la burguesía gallega en la polémica sobre el foro parece que no puede ser explicado de otra forma que por su apoyo tácito a las medidas que la revolución liberal había adoptado en Galicia. Medidas que, no hay que olvidarlo, permitieron, y en especial la desamortización, que la burguesía gallega se colocase entre los principales perceptores de rentas agrarias de Galicia ${ }^{14}$.

Para Colmeiro el foro imponía cuatro importantes obstáculos a la mejora de la agricultura ${ }^{15}$ :

1. La inexistencia de una garantía expresa de que fuesen respetados los derechos de los «utilitarios».

2. La irredimibilidad de la renta foral. Con lo que el foro se desviaba de la «naturaleza» de los censos, con los cuales (especialmente con la enfiteusis) tiene mil puntos de contacto.

3. La gran subdivisión, en «partecillas moleculares» del «dominio útil». Pese a la gran extensión de las tierras controladas por los «dominios directos» no se favorecía la agricultura de grandes extensiones y sí en cambio la excesiva partición de éstas.

4. El «entorpecimiento» causado por los laudemios al gravar la propiedad territorial y castigar las mejoras que hiciese el «utilitario».

Para solventar estas dificultades proponía la perpetuidad y redimibilidad de los foros. No entraba en los pormenores de una ley de redención que, a su juicio, debía de ser redactada tras consultar muy escrupulosamente los intereses de foristas y foreros. Los de los primeros facilitando al máximo la redención por medio de pagos fraccionados. Los de los foristas asegurándoles una indemnización que les compensase suficientemente ${ }^{16}$.

14 Cordero Torrón (1986).

${ }^{15}$ Colmeiro (1843), p. 44.

36 «Los remedios, en nuestra humilde opinión, más eficaces para cicatrizar heridas tan profundas, serían:

1. Obtener una declaración legal de perpetuidad a favor de los foros.

2. Obtener otra ley que los declarase redimibles. En los pormenores de la redención debieran consultarse muy escrupulosamente los intereses del forista y del forero: los de éste facilitándosela lo más posible, ora permitiéndole redimir por partes, ora dándole libertad de satisfacer el capital reintegrable en granos, si la pensión era frumentaria, etc. Los de aquél disponiendo la redención de manera que obtuviese una completa indemnización, por ejemplo, haciéndole ganar en capital lo que perdiese con no rescatarlo en dinero, o conforme fuese menor cada entrega al amortizarlo por fracciones. Bien columbramos dificultades no pequeñas al conciliar intereses, sino encontrados, a lo menos muy divergentes; pero al legislador toca vencerlos con su prudencia y sabiduría.» Colmeiro (1843), pp. 44 y 45 . 
El cuadro de la transformación lenta y gradual de la economía gallega que vislumbraba, se completaría con la instrucción pública, el desarrollo de la industria y el comercio, las mejoras en las vías de comunicación y la libertad del comercio interior $y$, aunque menguada por la existencia de alguna protección temporal, también del exterior ${ }^{17}$.

La crítica que efectúa del régimen foral en la Memoria volverá a repetirla, aunque de forma menos explícita, en el Tratado Elemental de Economía Politica Ecléptica y en sucesivas ediciones de los Principios de Economía Politica.

En su Tratado, publicado en 1845, tras señalar que la enfiteusis era el contrato agrario más ventajoso para el desarrollo de la agricultura, indicaba que:

«Para que el contrato enfitéutico produjese todos los beneficios de que es capaz, sería menester acomodar las dimensiones de cada labranza a las fuerzas productivas de una familia, porque si el enfiteuta poseyese menos tierra de labor, no saldría completamente de la condición precaria y miserable del obrero: y, si poseyese más, o las cultivaría mal, o las daría en subarriendo, cosa de muy pernicioso efecto sobre los progresos del cultivo" ${ }^{18}$.

Los obstáculos que se oponían a los beneficios de la enfiteusis y, por ello, del foro, eran: la inexistencia del capital suficiente para una explotación eficiente, debido a la reducida extensión de las explotaciones agrarias, y las posibles colisiones entre los «condueños» (del «directo» y del «útil»), derivadas de la mancomunidad de derechos. Los remedios serían: «acomodar las dimensiones de cada labranza» y «arreglar los intereses de los condueños para que la comunión sea un lazo, no un motivo de querellas». Para ello, se debía partir siempre de la idea de «consolidar el dominio, atrayendo el directo al útil, si conviniera desmembrar la propiedad, y procurando confundir el útil en el directo, si al contrario importase reorganizarla por medio de la concentración" ${ }^{19}$.

${ }^{17}$ Sobre la Instrucción pública, Colmeiro (1843), pp. 36 y ss.; sobre la industria, pp. 53 y ss.; sobre las vías de comunicación, p. 63, y sobre comercio, p. 64.

${ }_{18}$ Colmeiro (1845), tomo II, pp. 306 y 307.

19 Colmeiro (1845), pp. 307 y ss. En los Principios de Economía Politica, 1." ed., Colmeiro (1859), pp. 61 y 62, señalaba que: «La enfiteusis es un contrato ventajoso bajo el punto de vista económico, porque el canon se estipula en consideración al valor actual de la finca, no sufre alteración y crea derechos perpetuos." A esta reflexión le sigue, desde la segunda edición de los Principios..., una dura crítica de los foros. De forma invariable, desde la segunda hasta la cuarta edición de los Principios... añade al párrafo de la primera edición en la que se destacan las ventajas de la enfiteusis lo siguiente: «Los foros de 
Colmeiro no fue el único en defender la redimibilidad de los foros. En el mismo año en que Colmeiro publicaba su Memoria, también Manuel Ortiz de Zúñiga iba a pedir ante la Comisión de Códigos encargada de la redacción del Código Civil que fuesen declarados redimibles los foros al igual que los restantes tipos de censos ${ }^{20}$. Y en el proyecto de Código Civil de los moderados de 1851 el foro era considerado, al igual que la enfiteusis, como un gravamen perpetuo que podía ser redimido por los «utilitarios» abonando al dueño del «directo» lo que resultase de capitalizar las rentas ${ }^{21}$.

Tampoco fue Colmeiro el primer economista gallego en defender la división de los comunales. Pues existía desde el siglo xviri una tendencia mayoritaria entre los economistas gallegos favorable a un aprovechamiento privado de los comunales en una línea muy similar a la que fue defendida por Colmeiro 22 .

Pero Colmeiro, como dijimos al principio, sí fue el primer economista gallego en vislumbrar en su globalidad el conjunto de medidas que se deberían de adoptar para provocar un cambio en la "constitución agraria» $\mathrm{y}$, con ello, eliminar las causas del bloqueo del crecimiento agrario y de la economía en su conjunto, pues, en su opinión, el cambio en la estructura de la propiedad y el crecimiento de la producción agraria alimentarían un incremento de los intercambios comerciales y una expansión de las actividades industriales:

«Aliviada poco a poco la propiedad territorial de las enormes cargas que la abruman con tan exorbitantes pensiones y gravámenes a que está sujeta, mejoraría la condición del labrador: es decir, que el labrador sería más rico á lo sucesivo. Entretanto el antiguo forista, o invertía el capital reintegrado en descuajar y reducir á labor nuevas tierras para satisfacer las mayores demandas de subsistencias, consecuencia natural de un aumento necesario de población, o cedería un lugar en su ánimo al espíritu fabril para ganar en industria mucho más que pudiera perder en rentas territoriales. Y como el manantial más abundoso de la pobreza, no tanto es la escasez de productos como el

Galicia y Asturias son una degeneración de la enfiteusis cuya utilidad, en cuanto promovieron la reducción de los eriales, está fuera de toda controversia; pero hoy perjudican a la agricultura agobiada por pensiones irredimibles, empobrecida con un cultivo diminuto, y en fin muerta por la inmensa confusión de los derechos de dominio.» 2.3 ed., Colmeiro (1865), pp. 137 y 138; 3." ed., Colmeiro (1870), p. 126, y 4." ed., Colmeiro (1873), pp. 123 y 124.

${ }_{20}^{20}$ En Boletín Judicial de Galicia (1866), núm. 43.

21 García Goyena (1852), pp. 814 y ss.

22 Sobre los economistas ilustrados gallegos y sobre sus posiciones sobre los bienes comunales, véase Dopico (1978). 
grande desnivel en la producción, porque solo compra aquel que tiene que vender, se colije facilmente que mejorada la suerte del labrador se aseguraban al mismo tiempo las salidas ó el despacho de los productos fabriles. ... Asi iríamos asentando las bases de una íntima alianza entre las fortunas industriales y comerciales y la aristocracia territorial, y venciendo esa funesta fuerza de inercia que caracteriza á la casi totalidad de nuestros grandes propietarios.... ${ }^{23}$.

Como las medidas que proponía, Colmeiro se anticipó en treinta años a la primera ley de redención general de foros, que tendría muy escasos resultados ${ }^{24}$ y en cerca de ochenta años a la definitiva ley de redención general de foros de 1926 y la división de los comunales se iría efectuando tiempo después paulatina y espontáneamente por los campesinos gallegos ${ }^{25}$. Mientras tanto, las crisis de subsistencia y la necesidad de articular la economía gallega en el mercado nacional español ${ }^{26}$ hacen aflorar en la década de los sesenta del siglo xIx la polémica sobre la necesidad de un cambio en la estructura de la propiedad en Galicia, retomándose entonces las ideas que había expuesto Colmeiro en la Memoria: pero los intereses económicos de los perceptores de rentas forales, antiguos foristas y burguesía comercial adquirente de rentas forales en la desamortización, consiguieron neutralizar cualquier medida reformista ${ }^{27}$. Quedando, entonces, condicionado, el desarrollo económico de Galicia por los obstáculos que una antigua forma de propiedad oponía al desarrollo de las fuerzas productivas.

${ }^{23}$ Colmeiro (1843), pp. 47 y 48.

24 López Rodríguez (1985).

${ }^{25}$ Bouhier (1979).

${ }^{26}$ Rodríguez Galdó y Dopico (1981).

${ }^{27}$ Villares (1982). 


\section{BIBLIOGRAFIA}

Alonso Alvarez, L. (1986): Comercio colonial y crisis del Antiguo Régimen en Galicia (1778-1818), La Coruña.

Barreiro Mallón, B. (1973): La Jurisdicción del Xallas en el siglo XVIII. Población, sociedad y economía, Santiago.

Baletín Mercantil e Industrial de Galicia (1848), núm. 73.

BouhIER, A. (1979): La Galice. Essat geographique d'analyse et d'interpretation d'un vieux complexe agraire, La Roche-sur-You (Vendèe).

Carmona Badí, X., y Cordero Torrón, X. (1980): «Introducción á analise da Redención Foral de Mendizábal en Galicia», Revista Gallega de Estudios Agrarios, núm. 3.

Colmeiro Penido, M. (1843): Memoria sobre el modo más acertado de remediar los males inberentes a la estremada subdivisión de la propiedad territorial de Galicia, Santiago.

- (1845): Tratado elemental de economía política ecléctica, Madrid, 2 tomos.

- (1859): Principios de economía politica, 1. ${ }^{3}$ ed.

- (1865): Principios de economía política, 2." ed.

- (1870): Principios de economía política, 3. ${ }^{3}$ ed.

- (1873): Principios de economía política, $4 .^{2}$ ed.

Cordero Torrón, X. (1986): «La redención foral en la provincia de La Coruña ante el proceso desamortizador», en Desamortización y bacienda pública, Madrid.

Dopico, F. (1978): A Ilustración e a Sociedade Galega. A visión de Galicia dos economistas ilustrados, Vigo.

García Goyena, F. (1852): Concordancias, motivos y comentarios del Código Civil español, Madrid (reeditado en Zaragoza en 1974).

García Lombardero, J. (1973): La agricultura y el estancamiento económico de Galicia en la España del Antiguo Régimen, Madrid.

García Lombardero, J., y Dopico, F. (1976): «La renta de la tierra y la polémica por la renovación de los foros en los siglos xvir y xvin!", Hacienda Pública Española, núm. 38.

García Ormaechea, R. (1932): Supervivencias teudales en España. Estudio de legislación $y$ jurisprudencia sobre señoríos, Madrid.

Lasso Gaite, J. F. (1979): Crónica de la codificación española. Codificación civil, Madrid.

López Rodríguez, P. (1985): Campesinos propietarios. La redención de foros en la provincia de Lugo durante la I República, Lugo.

Moxo, S. (1965): La disolución del régimen señorial en España, Madrid.

Pérez García, J. M. (1979): Un modelo de sociedad del Antiguo Régimen en la Galicia costera, Santiago.

Rodríguez Galdó, M. X. (1981): “A polemica librecambismo-proteccionismo en Galicia a mediados do seculo xIX. Fontes para o seu estudio», Grial, núm. 73.

Rodríguez Galdó, M. X., y Dopico, F. (1981): Crisis agrarias y crecimiento económico en Galicia en el siglo XIX, La Coruña.

SAAvedRA, P. (1979): Economia rural antigua en la montaña lucense, Santiago.

- (1985): Economia, politica y sociedad en Galicia: la provincia de Mondoñedo, 1480 . 1830, Santiago.

Villares, R. (1982): La propiedad de la tierra en Galicia: 1500-1936, Madrid. 